\title{
Growth charts and prediction of abnormal growth what is known, what is not known and what is misunderstood
}

\author{
Anna Kajdy, Jan Modzelewski, Krzysztof Herman, \\ Katarzyna Muzyka-Placzynska, Michal Rabijewski \\ Department of Reproductive Health, Center of Postgraduate Medical Education, Poland
}

\begin{abstract}
Objectives: Assessment of fetal growth has an important effect on perinatal morbidity and mortality. To understand what tool to choose best for a given population a basic knowledge of how growth charts are developed and used has to be acquired. For this reason, this literature review was performed.

Material and methods: An extensive literature review aimed at identifying articles related to the development of growth assessment in both spectrums of abnormal fetal growth — large and small. The analyzed articles were chosen and presented to show both the historical aspects of growth assessment, current trends and future considerations.

Results: Identification of both large and small fetuses and neonates is equally crucial. Definitions and methodology vary worldwide and there is an ongoing discussion on the best tool to choose for a given population. An important part of the debate is how to differentiate between the physiologically small fetus and the truly growth restricted fetus who is at risk of perinatal complication. Similarly, the diagnosis of a large fetus is important in prevention of perinatal complications and surgical deliveries. Many clinical settings still lack growth standards.

Conclusions: Birthweight for gestational age charts are biased for weight in preterm birth. Prediction and management of outcome cannot be based solely on fetal size. Small is not the only problem, we have to think large as well. A common misunderstanding in clinical practice is not using uniform charts in defining growth.
\end{abstract}

Key words: growth charts; small for gestational age; large for gestational age; appropriate for gestational age; growth standard; growth reference

Ginekologia Polska 2019; 90, 12: 717-721

\section{INTRODUCTION}

Growth assessment is crucial for predicting fetal wellbeing. It cannot be properly defined without a tool that best fits a studied population. Numerous growth charts have been developed. Only a handful of countries have recommendations for detection and management of abnormal growth, but still many do not have such standards [1]. Views around the world vary, for growth charts can be based on data collected locally or internationally. Some believe that in optimal conditions growth is the same irrelevant of ethnicity, others believe that local standards work better. A third group believes that growth should be customized based on maternal characteristics, including ethnicity, parity, maternal height and weight [2]. To understand what tool to choose best for a given population a basic knowledge of how growth charts are developed and used has to be acquired. For this reason, we have performed this literature review.

\section{A historical perspective}

Historically a strong association between fetal size and perinatal mortality has always been recognized. In 1950 World Health Organization has established a definition of Low Birth Weight (LBW) in which infants weighing less than 2500 grams at birth were considered "premature" [3]. Unfortunately, such definition has failed to differentiate between those born too small because born too soon from those born small because of growth restriction. Lubchenco et al. developed the first weight-for-gestational age percentile chart. Basing on it they introduced a classification were infants below the $10^{\text {th }}$ percentile were named SGA 
- small-for-gestational-age, between $10-90^{\text {th }}$ percentile AGA - appropriate for gestational age and above the $90^{\text {th }}$ centile LGA — large for gestational age [4].

In the late 60 's two groups published cross-classifications of birthweight and gestational age. Yerushalmy et al. suggested that neonatal mortality was linked to birthweight and gestational age. In group 1, all weighing < 1500 grams had a reported neonatal mortality of 707.3/1000; group $2<37$ weeks, weighing 1501-2500 gram 104.7/1000, group 3 (> 37 wks, 1501-2500 grams) 32/1000, group 4 ( $<37$ wks. and $>2500$ grams) 13.7 per thousand and group $\mathrm{V}$ (> $37 \mathrm{wks}$ and > 2500 grams) 4.7/1000 [5]. A similar very complex classification was also developed by Lubchenco, which included 9 different risk groups [4]. This has led to the false assumption that weighing more than 2500 grams at term is enough. This association was predominately limited by the obvious inadequacy of pregnancy dating before the widespread development and use of early gestation ultrasound. The current perinatal mortality in different populations varies from 4-8/1000. Lowering these rates remains a constant challenge of perinatal medicine.

An important question remains how to define the optimal cut off point for SGA and FGR. Although often used interchangeably, these are two different entities. Different cut-offs have been proposed - the $3^{\text {rd }}, 5^{\text {th }}, 10^{\text {th }}$ and $15^{\text {th }}$ centiles. Predominantly the 10th centile has been used. In some countries use of -2 standard deviations dominates [6]. Another alternative is a reference based on relative risk, where a weight correlation with a 2-fold, 2,5-fold and 3-fold risk of neonatal death at each gestational age has been established [7]. Another methodology was built on an outcome-based standard which also incorporates differences between singleton and multiple gestations [8]. The latter study has also addressed the problem that not only small but also excess fetal and neonatal weight is a risk factor for abnormal outcome.

Via the development of ultrasound methods and pregnancy dating, it was soon established that the earlier growth charts were biased especially for preterm deliveries. So, a question followed what to base the estimation of size on. Many tools were developed over time which will be described in the lower sections of this article. Recently more attention is given to the other side of the growth spectrum. LGA and macrosomia are coming into the research scope.

The question therefore arises, what it means to achieve an optimal growth potential for it seems it is not the same as being the right size.

\section{Defining optimal weight}

Optimal growth reflects the quality of intrauterine life and is a predictor of neonatal outcome. It is affected by many factors: maternal, fetal and placental. Growth charts can be standard or reference and can be obtained based on newborn weight or prenatal ultrasound estimation of foetal weight [9]. Reference growth charts reflect how growth is in a given population while standard growth charts reflect how growth should be [10]. Therefore the first will include records with risk factors affecting growth, while the latter will represent growth in optimal conditions without influencing factors [9]. Undeniably to determine pathological growth both large and small, a gold-standard tool has to be established. The discussion is whether it is better done by local reference, global standardization or customization. WHO and Intergrowth are international attempts to develop such standards [11, 12]. The philosophy behind these projects is that in fetal life and up to the age of five growth is the same regardless of ethnicity, maternal or fetal predisposing factors [12]. On the other hand, one of the most commonly used newborn growth chart is the Fenton growth chart which is a metanalysis of local reference charts from different parts of the world [13]. Similarly, the Yudkin, Alexander, Lubchenco growth charts used for prenatal growth assessment are indeed reference charts derived from newborn birth weights [14].

Those birthweight-for-gestational-age charts have several limitations. First of all, accurate information on gestational age is needed for constructing them. In the case of early charts, from the beginning it was known that they were biased especially for preterm deliveries [4]. The rationale behind it was that preterm birth has a component of abnormal placentation which affects growth, therefore neonates born preterm are smaller than their peers that have continued to grow in utero. This is reflected when comparing with charts based on estimated fetal weight. Secondly the first birth-for-gestational age charts did not exclude pregnancies with pathologies affecting growth. Weiner and colleagues compared conventional charts with in utero charts showing this relationship and concluding that preterm infants may be affected by suboptimal growth [15]. This was later compared in studies by Secher and Ott $[16,17]$. In conclusion conventional charts classify a smaller proportion of FGR across gestational ages. The relationship between spontaneous preterm birth and birthweight is crucial to understanding its bias on reference charts. Morken and colleagues, examined the risk of spontaneous preterm birth among over 1 million births and found that both undergrowth and overgrowth are associated with early parturition $[(95 \% \mathrm{Cl} 1.1,1.2)$ vs $(95 \%$ Cl 1.5, 1.7)] [18].

The INTERGROWTH-21st Project was aimed at designing an international growth standard based on a description of how individuals in healthy communities grow regardless of ethnic origin. 60,000 participants have been enrolled. The eligibility criteria were designed to identify healthy populations, following current health recommendations and 
living in environments highly unlikely to constrain growth. It included 3 complementary components: a cross-sectional study of all newborns in eight study sites located in Europe, Asia, Africa and the Americas; a cohort of healthy women in a longitudinal component to monitor fetal growth with ultrasound scans every $5 \pm 1$ weeks from 9-14 weeks of gestation to birth. The cohort was then followed for two years to assess health, growth, and neurodevelopment. Finally, in the third component, the postnatal growth of prematurely born infants was monitored. The study concluded indeed that growth in optimal health conditions is the same regardless of country or ethnicity. Similarly, WHO has conducted a study of intrauterine growth standard following similar criteria as INTERGROWTH [12]. They followed 1,387 healthy women with unconstrained nutritional and social background. On the contrary, they have concluded that fetal growth showed variation between countries that to a lesser extent was affected by maternal size, parity and fetal sex. In conclusion the authors suggested that these charts are more suitable for international use. The observed differences should be included in adjustment of the growth standard for local clinical use to increase the diagnostic and predictive performance [11].

Nicolaides at al. created a birth-weight and estimated fetal weight chart for all babies at a given gestational age [19]. The main reason for such aim was, that the regular approach of deriving birth-weight charts is misleading, since a large proportion of babies born preterm arise from pathological pregnancy, while reference ranges of estimated fetal weight are representative of the whole population but are also subject to a percentage error. Two datasets were created. First comprised of 5163 paired measurements of estimated fetal weight and birth weight; ultrasound examinations were carried out at 22-43weeks' gestation and birth occurred within 2 days of the ultrasound examination. Second comprised of 95579 pregnancies with EFW obtained by routine ultrasonographic fetal biometry at certain time of pregnancy $(20+0$ to $23+6$ weeks, $31+0$ to $33+6$ weeks or $35+0$ to $36+6$ weeks). The study has demonstrated that a very high proportion of preterm births are SGA. Authors have established a birthweight chart for all babies at a given gestational age, including those still in utero. Birthweight and estimated fetal weight charts had a common median but differ in the levels of spread from the median [12].

Mikolajczyk et al. created a generic adaptable for local populations reference for fetal and birthweight. Using Hadlock's fetal-weight reference and Gardosi's notion of proportionality they created a weight reference for any local population adjustable to the mean birthweight at 40 weeks gestational age. The method was validated using data from Data the 2004-08WHO Global Survey on Maternal and Perinatal Health (237,025 births, 24 countries), They concluded that this system can be easily adapted to local populations and has a better ability to predict adverse perinatal outcomes than non-customized fetal-weight reference and is simpler to use than the individualized reference without loss of predictive ability. They stressed the financial burden of developing local charts, promoting their method for low income countries [20].

Customization of growth is a method developed by Gardosi et al. that uses coefficients for maternal race, parity, height, weight, smoking and fetal sex to determine optimal growth [21]. The coefficients are generated based on birth-weight-for-gestational age charts for a given population. Grow charts are generated separately for each fetus to reflect his/her individual growth potential. Customized growth standard was developed for UK population at first, but the idea of customization is gaining popularity and therefore customized growth charts are available for many other populations [2].

Different growth charts were compared in a retrospective head-to-head manner for abnormal growth detection and adverse outcome prediction. Most presented studies reveal that use of customized standard increased detection rate of SGA babes and therefore could help to predict adverse outcome [22-24]. Some authors on the other hand did not find any added value in using customized growth charts in given population [25].

Effect of customization of growth standard in LGA is a much less studied problem. Bukowski et al. made a case-control study of stillbirths. Authors found that being LGA was a significant risk factor of stillbirth, but this was visible only when using customized or ultrasound norms [26].

2014 Cochrane systematic review concluded that there are no good quality evidence papers to be included into review [25]. More recent meta-analysis of observational studies by Chiossi et al. concluded that customized growth charts performed better than population growth charts in identification of at risk SGA but not LGA babies [27].

\section{Definitions, detection and consequences}

The two main aims of assessing growth is to plan obstetrical interventions and to anticipate newborn needs at birth. Small for gestational age and large for gestational age are the opposite spectrums of abnormal growth. Fetal growth restriction and macrosomia are definitions reflecting pathologically abnormal growth that require subsequent monitoring and adequate management. Therefore, respectively they are not synonyms of SGA and LGA. SGA is a birth weight definition representing fetuses and newborns that are smaller than their counterparts at the same gestational age whereas LGA reflects fetuses that are larger. Macrosomia on the other hand by definition is estimated fetal weight, with most commonly accepted cutoffs of above 4000 or $4500 \mathrm{~g}$ [28]. 


\section{Table 1. Fetal growth restriction definition}

\section{FGR definition establish in Delphi protocol}

\begin{tabular}{l|l}
$\begin{array}{l}\text { Early FGR (GA }<32 \text { weeks): } \\
\text { Absence of congenital } \\
\text { anomalies }\end{array}$ & $\begin{array}{l}\text { Late FGR }(G A \geq 32 \text { weeks) } \\
\text { Absence of congenital } \\
\text { anomalies }\end{array}$ \\
$\begin{array}{l}\text { EFW or AC }<3^{\text {th }} \text { percentile } \\
\text { Or } \\
\text { UA-AEDF }\end{array}$ & EFW/AC $<3^{\text {th }}$ centile \\
Or & $\begin{array}{l}\text { Or } 2 \text { out of } 3 \text { : } \\
\text { EFW/AC }<10^{\text {th }} \text { centile }\end{array}$ \\
with: & EFW/AC crossing \\
UtA PI $>95^{\text {th }}$ centile & centiles $>2$ quartiles on growth \\
UA th $>95^{\text {th }}$ centile & $\begin{array}{l}\text { centiles } \\
\text { CPR }<5^{\text {th }} \text { centile or UA-PI }>95^{\text {th }} \\
\text { centile }\end{array}$
\end{tabular}

FGR — fetal growth restriction; EFW — estimated fetal weight; AC - abdominal circumference; UA AEDF — umbilical artery absent end diastolic flow; UtA PI — uterine artery pulsatility index; UA PI — umbilical artery pulsatility index; CPR - cerebro-placental ratio (55) by expert consensus, a definition for early and late fetal growth restriction (FGR

The primary aim of detection of large fetuses is prevention of stillbirth, shoulder dystocia, maternal peripartum hemorrhage, injuries of both mother and neonate, cesarean section as well risk of maternal diabetes and metabolic syndrome in childhood [29]. In recent study Caradoux et al. compared different strategies to predict macrosomia and LGA. Authors compared longitudinal growth assessment, abdominal circumference longitudinal growth assessment and third trimester cross-sectional evaluation. Longitudinal assessment used second trimester and third trimester growth velocity. Ultrasound scans and pregnancy outcomes of 2696 women were analyzed. Authors concluded that single assessment of abdominal circumference in third trimester performed better than longitudinal growth assessment [30].

If LGA or macrosomia are risk factors of stillbirth remains controversial. Study by Bukowski et al. showed that there is a significant relation between LGA and stillbirth if individualized growth charts are used. This relation was not visible when using population growth charts [26].

The concept of FGR except for smallness reflects also the placenta related underlying pathology. ACOG defines FGR as a fetus failing to reach its growth potential in absence of any pathological conditions or toxic factors. Because of different population standards there is no single optimal weight related definition for growth restriction. Some authors consider an infant to have FGR if birth weight falls below the $3^{\text {rd }}$ percentile or below two standard deviations of the mean for a given gestational age [31].Various definitions of fetal growth restriction based on population norms result in discrepancy in FGR diagnosis. The same infant maybe diagnosed on one growth chart as FGR but may be above the 10th percentile cutoff on another growth standard [32, 33]. In effect the reported incidence has a wide range from 4 to $8 \%$ in developed countries and up to $30 \%$ in developing countries [9]. The latest published definition of FGR is a result of implementing a Delphi protocol to reach an international consensus. 56 experts were asked questions about importance of ultrasound findings for growth restriction diagnosis. The final definitions are presented in Table 1. Although this definition is not a result of direct clinical investigation but an expert opinion it is most commonly used in research settings and management protocols [34]. The experts had agreed that fetal weight should not be based on customized standards. The reported incidence of LGA vary between studies from $4,6 \%$ to $15,9 \%$, depending on study population and growth chart used $[33,35]$.

\section{Conclusions and future considerations}

It is a well-established fact that assessing only fetal weight is not enough to predict outcome. There are several online tools that combine use of size and Doppler indices in management of SGA and its differentiation from FGR (Fetal Medicine Foundation and Fetal Medicina Barcelona Calculator). There are several ongoing trials for prediction and management of pregnancies complicated by overgrowth. Management tools such as those for SGA are yet to be established. Perhaps in future artificial intelligence will allow research to analyze the many combinations of factors that affect fetal growth and neonatal outcome (fetal, maternal, environmental and placental).

\section{What is known?}

Birthweight for gestational age charts are biased for weight in preterm birth.

A single size assessment does not reflect the growth potential. Local population standards perform better than global standards. Randomized trials of customization are needed for validation of its use in prevention of complications especially in preterm pregnancies.

\section{What is not known?}

Prediction and management of outcome cannot be based solely on fetal size; other factors have to be considered. Up to date not one management tool, recommendation nor standard has been proven to be superior.

\section{What is misunderstood?}

Small is not the only problem, we have to think large as well. A common misunderstanding in clinical practice is not using uniform charts in defining growth. Basing decision solely on fetal size, without considering other factors such as Doppler assessment can result in unnecessary interventions.

\section{REFERENCES}

1. McCowan LM, Figueras F, Anderson NH. Evidence-based national guidelines for the management of suspected fetal growth restriction: comparison, consensus, and controversy. Am J Obstet Gynecol. 2018; 
218(2S): S855-S868, doi: 10.1016/j.ajog.2017.12.004, indexed in Pubmed: 29422214

2. Gardosi J, Francis A, Turner S, et al. Customized growth charts: rationale, validation and clinical benefits. Am J Obstet Gynecol. 2018; 218(2S): S609-S618, doi: 10.1016/j.ajog.2017.12.011, indexed in Pubmed: 29422203.

3. World Health Organization. Expert group on Prematurity: final report. Technical Report Series, no. 27. Geneva, 17-21 April 1950. https://apps. who.int/iris/bitstream/handle/10665/38549/WHO_TRS_27_eng.pdf.

4. Battaglia FC, Lubchenco LO. A practical classification of newborn infants by weight and gestational age. J Pediatr. 1967; 71(2): 159-163, doi: 10.1016/s0022-3476(67)80066-0, indexed in Pubmed: 6029463.

5. Yerushalmy J. The classification of newborn infants by birth weight and gestational age. J Pediatr. 1967; 71(2): 164-172, doi: 10.1016/s00223476(67)80067-2, indexed in Pubmed: 6029464.

6. Romero R, Tarca AL. Fetal size standards to diagnose a small- or a large-for-gestational-age fetus. Am J Obstet Gynecol. 2018; 218(2S): S605-S607, doi: 10.1016/j.ajog.2017.12.217, indexed in Pubmed: 29422201.

7. Boulet SL, Alexander GR, Salihu HM, et al. Fetal growth risk curves: defining levels of fetal growth restriction by neonatal death risk. Am J Obstet Gynecol. 2006; 195(6): 1571-1577, doi: 10.1016/j.ajog.2006.03.069, indexed in Pubmed: 16769013.

8. Joseph KS, Fahey J, Platt RW, et al. An outcome-based approach for the creation of fetal growth standards: do singletons and twins need separate standards? Am J Epidemiol. 2009; 169(5): 616-624, doi: 10.1093/aje/kwn374, indexed in Pubmed: 19126584.

9. Wehbe M, Charafeddine L, Yunis K. Intrauterine Growth Standards. Handbook of Growth and Growth Monitoring in Health and Disease. 2011: 93-114, doi: 10.1007/978-1-4419-1795-9_6.

10. Hutcheon JA, Egeland GM, Morin L, et al. The predictive ability of conditional fetal growth percentiles. Paediatr Perinat Epidemiol. 2010; 24(2): 131-139, doi: 10.1111/j.1365-3016.2010.01101.x, indexed in Pubmed: 20415768.

11. Kiserud T, Piaggio G, Carroli G, et al. The World Health Organization Fetal Growth Charts: A Multinational Longitudinal Study of Ultrasound Biometric Measurements and Estimated Fetal Weight. PLoS Med. 2017; 14(1): e1002220, doi: 10.1371/journal.pmed.1002220, indexed in Pubmed: 28118360.

12. Garza C. The INTERGROWTH-21st project and the multicenter growth reference study: enhanced opportunities for monitoring growth from early pregnancy to 5 years of age. Breastfeed Med. 2014; 9(7): 341-344, doi: 10.1089/bfm.2014.0106, indexed in Pubmed: 25211458.

13. Fenton TR, Kim JH. A systematic review and meta-analysis to revise the Fenton growth chart for preterm infants. BMC Pediatr. 2013; 13: 59, doi: 10.1186/1471-2431-13-59, indexed in Pubmed: 23601190.

14. Yudkin PL, Aboualfa M, Eyre JA, et al. New birthweight and head circumference centiles for gestational ages 24 to 42 weeks. Early Hum Dev. 1987; 15(1): 45-52, doi: 10.1016/0378-3782(87)90099-5, indexed in Pubmed: 3816638.

15. Weiner $C P$, Sabbagha RE, Vaisrub N, et al. A hypothetical model suggesting suboptimal intrauterine growth in infants delivered preterm. Obstet Gynecol. 1985; 65(3): 323-326, indexed in Pubmed: 3883260.

16. Secher NJ, Kern Hansen $\mathrm{P}$, Thomsen BL, et al. Growth retardation in preterm infants. Br J Obstet Gynaecol. 1987; 94(2): 115-120, doi: 10.1111/j.1471-0528.1987.tb02336.x, indexed in Pubmed: 3548805.

17. Ott WJ. Intrauterine growth retardation and preterm delivery. Am J Obstet Gynecol. 1993; 168(6 Pt 1): 1710-1717, doi: 10.1016/00029378(93)90681-8, indexed in Pubmed: 8317512.

18. Morken NH, Källen K, Jacobsson Bo. Fetal growth and onset of delivery: a nationwide population-based study of preterm infants. Am J Obstet Gynecol. 2006; 195(1): 154-161, doi: 10.1016/j.ajog.2006.01.019, indexed in Pubmed: 16813752.

19. Nicolaides $K H$, Wright $D$, Syngelaki $A$, et al. Fetal Medicine Foundation fetal and neonatal population weight charts. Ultrasound Obstet Gynecol. 2018; 52(1): 44-51, doi: 10.1002/uog.19073, indexed in Pubmed: 29696704.

20. Mikolajczyk RT, Zhang J, Betran AP, et al. A global reference for fetal-weight and birthweight percentiles. Lancet. 2011; 377(9780):
1855-1861, doi: 10.1016/S0140-6736(11)60364-4, indexed in Pubmed: 21621717.

21. Gardosi J, Mongelli M, Wilcox M, et al. An adjustable fetal weight standard. Ultrasound Obstet Gynecol. 1995; 6(3): 168-174, doi: 10.1046/j.146 9-0705.1995.06030168.x, indexed in Pubmed: 8521065.

22. Ego A, Subtil D, Grange $G$, et al. Customized versus population-based birth weight standards for identifying growth restricted infants: a French multicenter study. Am J Obstet Gynecol. 2006; 194(4): 1042-1049, doi: 10.1016/j.ajog.2005.10.816, indexed in Pubmed: 16580294.

23. Odibo AO, Nwabuobi $C$, Odibo L, et al. Customized fetal growth standard compared with the INTERGROWTH-21st century standard at predicting small-for-gestational-age neonates. Acta Obstet Gynecol Scand. 2018; 97(11): 1381-1387, doi: 10.1111/aogs.13394, indexed in Pubmed: 29878301.

24. Anderson NH, Sadler LC, McKinlay CJD, et al. INTERGROWTH-21st vs customized birthweight standards for identification of perinatal mortality and morbidity. Am J Obstet Gynecol. 2016; 214(4): 509.e1-509.e7, doi: 10.1016/j.ajog.2015.10.931, indexed in Pubmed: 26546850.

25. Carberry AE, Gordon A, Bond DM, et al. Customised versus population-based growth charts as a screening tool for detecting small for gestational age infants in low-risk pregnant women. Cochrane Database Syst Rev. 2014(5): CD008549, doi: 10.1002/14651858.CD008549.pub3, indexed in Pubmed: 24830409.

26. Bukowski R, Hansen NI, Willinger M, et al. Eunice Kennedy Shriver National Institute of Child Health and Human Development Stillbirth Collaborative Research Network. Fetal growth and risk of stillbirth: a population-based case-control study. PLoS Med. 2014; 11(4): e1001633, doi: 10.1371/journal.pmed.1001633, indexed in Pubmed: 24755550.

27. Chiossi G, Pedroza C, Costantine MM, et al. Customized vs population-based growth charts to identify neonates at risk of adverse outcome: systematic review and Bayesian meta-analysis of observational studies. Ultrasound Obstet Gynecol. 2017; 50(2): 156-166, doi: 10.1002/uog.17381, indexed in Pubmed: 27935148.

28. American College of Obstetricians and Gynecologists' Committee on Practice Bulletins-Obstetrics. Practice Bulletin No. 173: Fetal Macrosomia. Obstet Gynecol. 2016; 128(5): e195-e209, doi: 10.1097/AOG.0000000000001767, indexed in Pubmed: 27776071.

29. Beta J, Khan N, Khalil A, et al. Maternal and neonatal complications of fetal macrosomia: systematic review and meta-analysis. Ultrasound Obstet Gynecol. 2019; 54(3): 308-318, doi: 10.1002/uog.20279, indexed in Pubmed: 30938004.

30. Caradeux J, Eixarch E, Mazarico E, et al. Second- to Third-Trimester Longitudinal Growth Assessment for the Prediction of Largeness for Gestational Age and Macrosomia in an Unselected Population. Fetal Diagn Ther. 2018; 43(4): 284-290, doi: 10.1159/000477460, indexed in Pubmed: 28719900.

31. Usher R, McLean F. Intrauterine growth of live-born Caucasian infants at sea level: standards obtained from measurements in 7 dimensions of infants born between 25 and 44 weeks of gestation. J Pediatr. 1969; 74(6): 901-910, doi: 10.1016/s0022-3476(69)80224-6, indexed in Pubmed: 5781799.

32. Carberry AE, Gordon A, Bond DM, et al. Customised versus population-based growth charts as a screening tool for detecting small for gestational age infants in low-risk pregnant women. Cochrane Database Syst Rev. 2014(5): CD008549, doi: 10.1002/14651858.CD008549.pub3, indexed in Pubmed: 24830409.

33. Sjaarda LA, Albert PS, Mumford SL, et al. Customized large-for-gestational-age birthweight at term and the association with adverse perinatal outcomes. Am J Obstet Gynecol. 2014; 210(1): 63.e1-63.e11, doi: 10.1016/j.ajog.2013.09.006, indexed in Pubmed: 24035985.

34. Gordijn SJ, Beune IM, Thilaganathan B, et al. Consensus definition of fetal growth restriction: a Delphi procedure. Ultrasound Obstet Gynecol. 2016; 48(3): 333-339, doi: 10.1002/uog.15884, indexed in Pubmed: 26909664.

35. Pritchard N, Lindquist A, Hiscock R, et al. Customised growth charts in large-for-gestational-age infants and the association with emergency caesarean section rate. Aust N Z J Obstet Gynaecol. 2019; 59(3): 380-386, doi: 10.1111/ajo.12868, indexed in Pubmed: 30088673. 